\title{
Behavioral and Anatomical Correlates of Chronic Episodic Hypoxia during Sleep in the Rat
}

\author{
David Gozal, ${ }^{1}$ Jill M. Daniel, ${ }^{2}$ and Gary P. Dohanich² \\ ${ }^{1}$ Kosair Children's Hospital Research Institute, Departments of Pediatrics, Pharmacology, and Toxicology, University of \\ Louisville School of Medicine, Louisville, Kentucky 40202, and 2Department of Psychology, Tulane University, New \\ Orleans, Louisiana 70118
}

The role played by chronic episodic hypoxia (EHYP) in the neurocognitive morbidity of obstructive sleep apnea (OSA) is unknown. Sleep recordings, Morris water maze experiments, and immunohistochemistry for NMDA NR1 glutamate receptor, c-fos protein, and apoptosis [nuclear immunoreactivity for single-stranded DNA and terminal deoxynucleotidyl transferase-mediated biotinylated UTP nick end labeling assay] were conducted in EHYP-exposed Sprague Dawley male rats. Exposures consisted of up to14 d in an environmental chamber in which $\mathrm{O}_{2}$ concentrations were cycled between 10 and $21 \%$ every $90 \mathrm{sec}$ or 30 min during $12 \mathrm{hr}$ of daylight. For the remaining $12 \mathrm{hr}$, EHYP rats breathed room air, while controls spent $14 \mathrm{~d}$ in room air. Although EHYP induced significant disruption of sleep architecture during the initial day of exposure, sleep patterns normalized thereafter. Marked increases in apoptosis occurred in the CA1 hippocampal region (sevenfold) and cortex (Cx; eightfold) after 1-2 d of EHYP but not in CA3 and were followed by decreases toward normoxic levels by $14 \mathrm{~d}$. Double labeling for NMDA NR1 and c-fos revealed marked architectural disorganization in CA1 and $\mathrm{Cx}$ with increases in c-fos over time. Rats exposed to EHYP displayed significantly longer escape latencies and swim path lengths to escape a hidden platform during 12 training trials given over $2 \mathrm{~d}$. Differences in the performances of EHYP and control rats, although reduced, persisted after $14 \mathrm{~d}$ of recovery. We conclude that EHYP is associated with marked cellular changes over time within neural regions associated with cognitive functions. Furthermore, EHYP impaired performance during acquisition of a cognitive spatial task without affecting sensorimotor function. Such changes may underlie components of the learning and memory impairments found in OSA.

Key words: sleep; apoptosis; intermittent hypoxia; immediate early genes; glutamate receptors; obstructive sleep apnea; cognitive impairment; memory; water maze
The clinical syndrome of obstructive sleep apnea (OSA), a condition characterized by repeated episodes of upper airway obstruction during sleep, affects $\sim 5 \%$ of the general population (Partinen and Telakivi, 1992; Redline et al., 1994; National Heart, Lung, Blood Institute Working Group on Sleep Apnea, 1996). The intermittent upper airway obstruction during sleep leads to episodic hypoxia, which may be severe with nadir hemoglobin oxygen saturations frequently reaching $50-60 \%$. In addition, periodic alveolar hypoventilation and repeated arousal either behavioral or electroencephalographic occur leading to sleep fragmentation and deprivation.

The major neurocognitive manifestations of OSA include excessive daytime sleepiness (Roehrs et al., 1995), personality and psychosocial maladjustment patterns, and mental impairment in terms of thinking, perception, memory, communication, or the ability to learn new information (Kales et al., 1985; Gozal, 1998). The relative contributions of sleep fragmentation and deprivation and those of episodic hypoxemia to the neurocognitive deficits exhibited by OSA patients are unclear and cannot be elucidated in humans for obvious ethical reasons (Berry et al., 1986; Bedard et al., 1991; Morisson et al., 1998). The available correlational studies of physiological disturbances during sleep and cognitive

\footnotetext{
Received Sept. 26, 2000; revised Dec. 13, 2000; accepted Jan. 11, 2001.

This study was supported by National Institutes of Health Grants HL-63912 and HL-65270 and by American Heart Association Grant AHA-0050442N. The technical assistance of Ying-Dan Xue and Ken Brittain is greatly appreciated.

Correspondence should be addressed to Dr. David Gozal, Kosair Children's Hospital Research Institute, University of Louisville, 570 South Preston Street, Suite 321, Louisville, KY 40202. E-mail: d0goza01@gwise.louisville.edu.

Copyright (C) 2001 Society for Neuroscience 0270-6474/01/212442-09\$15.00/0
}

deficits in humans further suggest that reductions in general intellectual measures and executive and psychomotor tasks are primarily attributable to the severity of hypoxemia, whereas other attention and memory deficits seem to be related to the impaired vigilance induced by sleep deprivation and fragmentation (Bedard et al., 1991). However, the latter may not be the only factor associated with excessive daytime sleepiness, because the ability to maintain wakefulness was markedly disturbed in 322 OSA patients and was inversely correlated to the degree of both sleep fragmentation and nighttime hypoxemia (Poceta et al., 1992).

In the present study, we aimed to develop an animal model in which the neurobehavioral effects of episodic hypoxia could be assessed in the absence of significant sleep fragmentation. We hereby demonstrate that exposure to episodic hypoxia during the usual resting period of young adult rats elicits both substantial region-selective neuronal loss and functional deterioration in the absence of sleep deprivation.

\section{MATERIALS AND METHODS}

Young adult Sprague Dawley rats (45-50 d of age) were used for all experiments. The experimental protocols were approved by the Institutional Animal Use and Care Committee and are in close agreement with the National Institutes of Health guide for the care and use of laboratory animals. All efforts were made to minimize animal suffering, to reduce the number of animals used, and to use alternatives to in vivo techniques.

Episodic hypoxia. Animals sojourned for $1 \mathrm{~d}(n=8), 2 \mathrm{~d}(n=8), 7 \mathrm{~d}$ $(n=8)$, and $14 \mathrm{~d}(n=32)$ in four identical commercially designed chambers $(30 \times 20 \times 20$ inches; Oxycycler model A44XO; Reming Bioinstruments, Redfield, NY) that can accommodate six rats each; the chambers were operated under a $12 \mathrm{hr}$ light/dark cycle (6:00 A.M.-6:00 P.M.). Gas was circulated around each of the chambers, attached tubing, 
and other units at $60 \mathrm{l} / \mathrm{min}$ (i.e., one complete change per $10 \mathrm{sec}$ ). The $\mathrm{O}_{2}$ concentration was continuously measured by an $\mathrm{O}_{2}$ analyzer and was changed throughout the $12 \mathrm{hr}$ of light time by a computerized system controlling the gas valve outlets, such that the moment-to-moment desired oxygen concentration of the chamber was programmed and adjusted automatically. Deviations from the desired concentration were corrected by addition of $\mathrm{N}_{2}$ or $\mathrm{O}_{2}$ through solenoid valves. For the remaining $12 \mathrm{hr}$ of nighttime, oxygen concentrations were kept at $21 \%$. Ambient $\mathrm{CO}_{2}$ in the chamber was periodically monitored and maintained at $<0.01 \%$ by adjusting overall chamber basal ventilation. The gas was also circulated through a molecular sieve (type 3A; Scientific Instrument Services, Ringoes, NJ) to remove ammonia. Humidity was measured and maintained at $40-50 \%$ by circulating the gas through a freezer and silica gel. Ambient temperature was kept at $22-24^{\circ} \mathrm{C}$.

Two episodic hypoxia (EHYP) profiles were used and consisted of alternating room air and $10 \%$ oxygen either every $90 \mathrm{sec}$ or every $30 \mathrm{~min}$ during the light phase. Because findings were nearly identical after both EHYP profiles, results were merged for presentation purposes. Time point-matched control animals were exposed for $1 \mathrm{~d}(n=8), 2 \mathrm{~d}(n=8)$, $7 \mathrm{~d}(n=8)$, and $14 \mathrm{~d}(n=27)$ to circulating normoxic gas in one of the four chambers.

Surgical and sleep-recording procedures. Under general anesthesia (pentobarbital, $50 \mathrm{mg} / \mathrm{kg}$, i.p.), six animals were implanted with cortical EEG and nuchal electromyographic (EMG) electrodes as described by Krueger et al. (1993). Briefly, EEG electrodes were placed over the frontal and parietal cortices, and insulated leads from the EEG and EMG electrodes were pulled under the skin to a Teflon base (Plastics One, Roanoke, VA) and cemented to the skull with dental cement (Hygienic Repair Resin, Akron, OH). A 1 week recovery period was allowed by placing the animals in individual cages within the intermittent hypoxia exposure chambers, after which experimental recordings were initiated. Analog signals from the EEG and EMG electrodes were displayed on a screen, digitized in $10 \mathrm{sec}$ epochs, and stored onto a Macintosh Personal Computer System at a $125 \mathrm{~Hz}$ sampling frequency using MacLab Digital Acquisition Software (AD Instruments, Castle Hill, Australia). EMG activity served as an aid for determining the vigilance states and was not further analyzed. The EEG was filtered below $0.1 \mathrm{~Hz}$ and above $40 \mathrm{~Hz}$. Vigilance states corresponding to wakefulness, rapid eye movement sleep (REMS), and non-REMS (NREMS) were determined in the $10 \mathrm{sec}$ epochs using previously validated criteria (Timo-Iaria et al., 1970; Franken et al., 1991; Frank and Heller, 1997) as follows: NREMS, highamplitude EEG slow waves and low-level EMG activity; REMS, highly regular $\theta$ activity in the EEG and a general lack of body movements with occasional twitches; and wakefulness, low-amplitude, fast EEG activity and high EMG activity. Time spent in each vigilance state was calculated for $2 \mathrm{hr}$ intervals. Group averages were calculated hourly and also for the entire dark and light periods.

Measurement of blood gas values. Arterial blood samples were obtained from an implanted arterial catheter in four additional rats. After withdrawal of 75-100 $\mu \mathrm{l}$ of blood in the dead space of the catheter, another $150 \mu \mathrm{l}$ was sampled for immediate analysis of $\mathrm{pO}_{2}, \mathrm{pCO}_{2}$, and $\mathrm{pH}$ with a blood gas analyzer (model 178; Ciba Corning). Measurements were performed during normoxia and at the end of the $90 \mathrm{sec}$ hypoxic cycle.

Morris water maze. The Morris water maze consisted of a circular pool, $1.8 \mathrm{~m}$ in diameter and $0.6 \mathrm{~m}$ in height, filled to a level of $35 \mathrm{~cm}$ with water maintained at a temperature of $27^{\circ} \mathrm{C}$ (Morris, 1981, 1984, 1989; Morris et al., 1986). Pool water was made opaque by addition of $150 \mathrm{ml}$ of nontoxic white tempera paint. A Plexiglas escape platform (12 cm in diameter) was positioned $2 \mathrm{~cm}$ below the water surface. Extramaze cues surrounding the maze were fixed at specific locations and were visible to the rats while in the maze. Maze performance was recorded by a video camera suspended above the maze and interfaced with a video tracking system (HVS Imaging, Hampton, UK). Albino rats used in the experiments were temporarily tattooed with a black mark to allow video tracking.

Place learning was assessed on eight place-training trials on the first training day and four place-training trials on the second training day. Each male was placed into the pool from quasirandom start points and allowed a maximum of $90 \mathrm{sec}$ to escape to the platform where he remained for $15 \mathrm{sec}$. Rats that failed to escape were led to the platform. The position of the platform remained constant across trials. Trials were separated by $120 \mathrm{sec}$. Performance was assessed by two measures, mean escape latencies and swim path distances. After the final place-training trial on the second training day, the platform was removed for a $60 \mathrm{sec}$ probe trial. Time spent in each of the four quadrants of the pool and the number of target crossings over the previous location of the platform were recorded. After the probe trial, visual discrimination was assessed on two cued trials. Each male was placed into the pool from quasirandom start points and allowed a maximum of $90 \mathrm{sec}$ to escape to a platform marked by a small flag. Performance was assessed by two measures, mean escape latencies and swim path distances. To test the recovery from EHYP, animals were retested in the water maze with the hidden platform moved to a different quadrant $14 \mathrm{~d}$ after the termination of intermittent hypoxia exposures. Place learning was assessed on eight training trials. Two separate sets of animals were evaluated at different times under the same paradigm. The only difference between the two replications of the experiment was a change from a black pool to a white pool.

Immunohistochemistry. Rats were anesthetized with pentobarbital (50 $\mathrm{mg} / \mathrm{kg}$, i.p.) and perfused transcardially with $200 \mathrm{ml}$ of PBS at an ambient temperature and then with $2.5 \%$ paraformaldehyde in cold PBS containing $5 \%$ sucrose, $\mathrm{pH}$ 7.4. The brain was removed immediately from the skull after perfusion and placed overnight in a fixative containing $1 \%$ paraformaldehyde in PBS and $30 \%$ sucrose at $4^{\circ} \mathrm{C}$. Coronal sections $(40$ $\mu \mathrm{m})$ were cut on a freezing microtome and divided into two series. One series was stained for Nissl substance with thionine, and the other was processed immunohistochemically. Sections were washed extensively in PBS and incubated in $0.4 \%$ Triton X-100 in PBS containing $1.5 \%$ normal goat serum (Vector Laboratories, Burlingame, CA) for $1 \mathrm{hr}$. Sections were then serially incubated with anti-c-fos (Santa Cruz Biotechnology, Santa Cruz, CA; catalog \#sc-52; 1:10,000 dilution) and with an antibody to the NR1 NMDA glutamate receptor subunit raised against a synthetic peptide LQNQKDTVLPRRAIEREEGQLQLCSRHRE corresponding to the $\mathrm{C}$ terminal of the rat NMDA receptor subunit (Chemicon, Temecula, CA; catalog \#AB1516; 1:2500) (Petralia et al., 1994), as described previously (Gozal et al., 1999). Adjacent sections were also processed for assessment of glial fibrillary acid protein (GFAP) using a commercially available antibody (Dako, Carpinteria, CA; catalog \#Z0334; 1:10,000).

For assessment of apoptosis, detection of DNA breaks by terminal deoxynucleotidyl transferase-mediated biotinylated UTP nick end labeling (TUNEL) was performed using a commercially available kit (Apoptag; Trevigen) in $5 \mu \mathrm{m}$ sections. In addition, the presence of singlestranded DNA as a marker for apoptosis was assessed using a monoclonal antibody in $30 \mu \mathrm{m}$ sections (Chemicon; catalog \#MAB 3299; 1:500). This approach has been shown to stain apoptotic cells specifically without staining necrotic cells, whereas TUNEL may stain both apoptotic and necrotic cells (Frankfurt et al., 1996).

After the primary antibody reaction was completed, the sections were then washed extensively in PBS, incubated in biotinylated anti-rabbit $\operatorname{IgG}$ (Vector Laboratories) diluted in $0.4 \%$ Triton X-100 in PBS for $1 \mathrm{hr}$, washed three times in PBS, incubated for $1 \mathrm{hr}$ in avidin-biotinylated horseradish peroxidase (Vectastain Elite kit; Vector Laboratories) diluted in $0.4 \%$ Triton X-100 in PBS, rinsed three times in Tris, $\mathrm{pH} 7.6$, and incubated in $50 \mathrm{mg} / \mathrm{ml}$ diaminobenzidine tetrahydrochloride (Sigma, St.

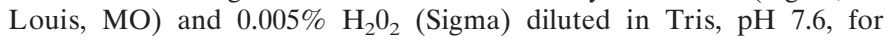
variable intervals until appropriate staining was achieved. The reaction was stopped in PBS, and the sections were mounted from sodium acetate onto slides coated with gelatin chrom-alum. For c-fos and glutamate receptor experiments, the resulting double-labeled neurons are easy to identify because c-fos is localized to the nucleus, whereas the glutamate receptor marker is found in the cytoplasm or plasma membrane. Control experiments were always done to determine whether the primary or secondary antibodies produced false-positive results.

Sections were assessed using a light microscope, and the distribution and number of cells containing the particular immunoreactivity were indicated on camera lucida drawings and maps of the hippocampus and cortex using the atlases by Paxinos and Watson (1986). The cytoarchitectural boundaries of the various hippocampal subregions and cortical layers were defined by superimposing the adjacent thionine-stained sections with the camera lucida drawings. We primarily analyzed the cellular patterning within the CA1 and CA3 subregions as well as the cortical layers encompassed within $-2.30 \mathrm{~mm}$ to $-4.80 \mathrm{~mm}$ from bregma using every sixth section. In general, 20 sections were assessed for every animal, and the data were tabulated and expressed as the number of observations per 100 or 1000 counted cells as appropriate. Changes across treatments or time points were compared using ANOVA procedures followed by Newman-Keuls post hoc tests. A $p$ value of $<0.05$ was considered to achieve statistical significance. 


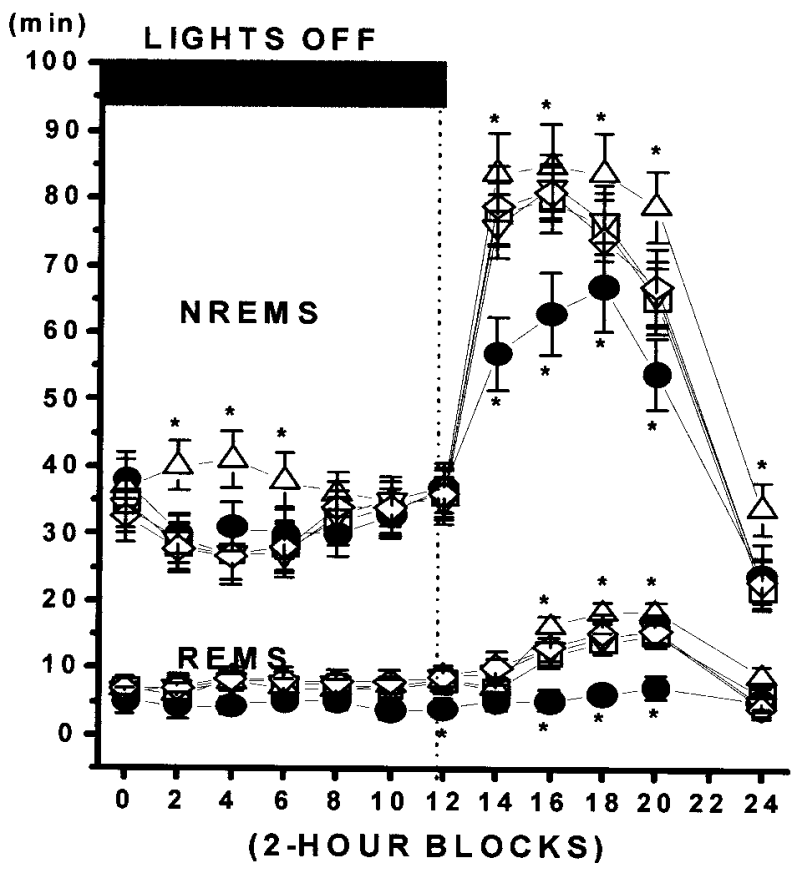

Figure 1. Mean duration of REMS and NREMS expressed in minutes per $2 \mathrm{hr}$ blocks and spanning over the circadian cycle in six rats during baseline conditions $(\square)$ and during day $1(\bullet)$, day $2(\Delta)$, day $7(\nabla)$, and day $14(\diamond)$ of EHYP exposure. Significant decreases in REMS and NREMS (indicated as *) occurred during day 1 ( $p<0.001$, ANOVA vs baseline), whereas significant increases in REMS and NREMS emerged during day 2 ( $p<0.002$, ANOVA vs day $1 ; p<0.03$, ANOVA vs baseline). The filled horizontal bar indicates the $12 \mathrm{hr}$ lights-off period. The vertical dashed line indicates the separation between lights-off and lights-on period.

\section{RESULTS}

\section{Gas exchange and sleep characteristics}

In four rats, arterial $\mathrm{pO}_{2}$ decreased from $89.4 \pm 3.2 \mathrm{mmHg}$ in room air to $44.2 \pm 2.2 \mathrm{mmHg}$ at the end of the 1st hypoxic cycle and decreased further to $36.7 \pm 2.1 \mathrm{mmHg}$ after the 10th hypoxic cycle with no further changes thereafter. Arterial $\mathrm{pCO}_{2}$ was $35.7 \pm 2.4 \mathrm{mmHg}$ in room air and decreased to $30.5 \pm 1.7$ and $26.7 \pm 1.4 \mathrm{mmHg}$ at the 1 st and 10th cycles, respectively, with no additional changes in subsequent cycles. Arterial $\mathrm{pH}$ was $7.36 \pm 0.02,7.46 \pm 0.03$, and $7.48 \pm 0.03$ in room air and at the end of the 1st and 10th cycles, respectively.

For sleep recordings in six rats, control conditions were considered to be present when $\sim 200 \mathrm{~min}$ of NREM and $30 \mathrm{~min}$ of REM sleep were consistently recorded during the $12 \mathrm{hr}$ dark period, indicative of acclimatization to the chamber environment. This process required 2-3 d in all but one rat who required $4 \mathrm{~d}$. After initiation of EHYP, disruptions in sleep architecture occurred during the initial $24 \mathrm{hr}$ EHYP epoch. Indeed, significant decreases in overall sleep duration that equally affected both NREM and REM sleep occurred (Fig. 1). During the second day of EHYP, a marked increase in NREM and REM sleep durations occurred, indicative of rebound sleep recovery. From days 3-14 of EHYP, the sleep characteristics returned to values similar to baseline. Thus, the EHYP protocol used in these experiments did not lead to sleep deprivation or fragmentation beyond the first day of exposure.

\section{Morris water maze}

Results from two replications of the water maze experiments were almost identical. Therefore the data were combined for analyses. During place training, the ability of rats to learn the location of a fixed hidden platform using extramaze spatial cues was determined. Figure $2 A$ illustrates that male rats $(n=18)$ exposed to $14 \mathrm{~d}$ of EHYP had more difficulty learning the location of the hidden platform than did control males $(n=19)$ across $2 \mathrm{~d}$ of place-training trials in the water maze. To assess performance during place training, escape latencies and swim path lengths were analyzed by two-way repeated measures ANOVA (with the treatment and two-trial block as factors and repeated measures on the block), followed by one-way ANOVA between treatments for each block. Rats exposed to $14 \mathrm{~d}$ of EHYP displayed significantly longer escape latencies $\left[F_{(1,35)}=\right.$ 8.14; $p<0.01$; Fig. $2 A]$ and path lengths $\left[F_{(1,35)}=7.53 ; p<0.01\right.$; Fig. $2 B]$ over the eight place-training trials on day 1 . There were significant block effects for escape latencies $\left[F_{(3,105)}=27.79 ; p<\right.$ 0 . 0001] and for path lengths $\left[F_{(3,105)}=11.00 ; p<0.0001\right]$, indicating that the performance of both groups improved over time. There were no significant treatment $\times$ block interactions. There were no differences in performance between treatment groups on the first block of day 1 because males from both groups learned the location of the platform. Rats exposed to $14 \mathrm{~d}$ of EHYP displayed significantly longer escape latencies and path lengths than did control animals on blocks 2,3 , and $4(p<0.05)$. Animals exposed to EHYP also displayed significantly longer escape latencies $\left[F_{(1,29)}=23.58 ; p<0.001\right.$; Fig. 2] and path lengths $\left[F_{(1,29)}=17.46 ; p<0.001\right.$; Fig. $\left.2 A, B\right]$ over the four place-training trials on day $2(n=15-16$; three rats from each group were killed after testing on day 1 for biochemical analyses). There were significant block effects for escape latencies $\left[F_{(1,29)}=\right.$ 7.21; $p<0.001]$ and for path lengths $\left[F_{(1,29)}=5.07 ; p<0.01\right]$. There were no significant treatment $\times$ block interactions. Significant differences $(p<0.05)$ were revealed between the performances of the groups on both blocks for escape latencies and path lengths.

Additionally, there were no significant differences between the performance of groups during two cued trials with a marked platform that were conducted to assess possible sensorimotor and motivational deficits (Fig. 2C). Escape latency and path length data were analyzed by two-way repeated measures ANOVA (with the treatment and trial as factors and repeated measures on the trial).

Figure $2 D$ illustrates that when animals were retested on the water maze with the platform moved to a new location $14 \mathrm{~d}$ after the termination of treatments, males exposed to EHYP continued to display impaired performance over eight training trials compared with males exposed to normoxia. Escape latency and swim path length data were analyzed by two-way ANOVA (treatment $\times$ two-trial block, with repeated measures on the block), followed by one-way ANOVA between treatments for each block. Rats exposed to EHYP $(n=13)$ displayed significantly longer escape latencies $\left[F_{(1,24)}=6.62 ; p<0.01 ;\right.$ Fig. $2 D$, left $]$ and path lengths $\left[F_{(1,24)}=6.59 ; p<0.01\right.$; Fig. $2 D$, right $]$ over the eight place-training trials than did males exposed to normoxia $(n=13)$. There were significant block effects for escape latencies $\left[F_{(3,72)}=\right.$ $18.60 ; p<0$. 0001] and for path lengths $\left[F_{(3,72)}=15.00 ; p<\right.$ $0.0001]$, indicating that the performance of both groups improved over time. There were no significant treatment $\times$ block interactions. Rats exposed to EHYP performed more poorly on blocks 1,2 , and 4. 

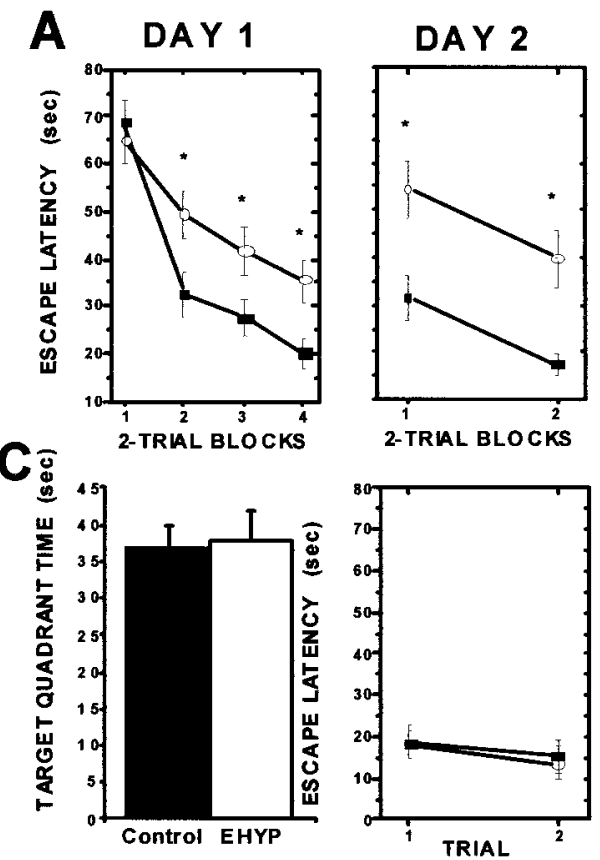

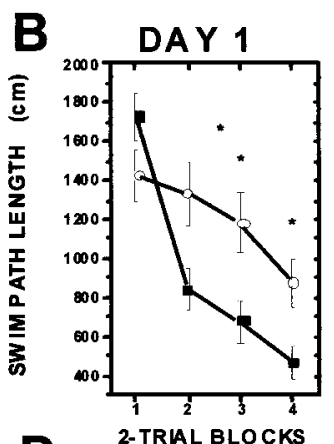

D

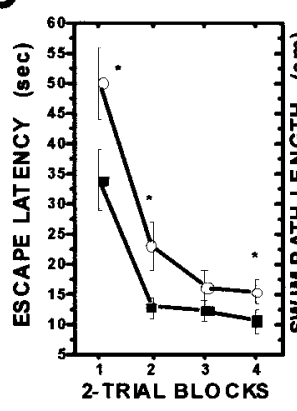

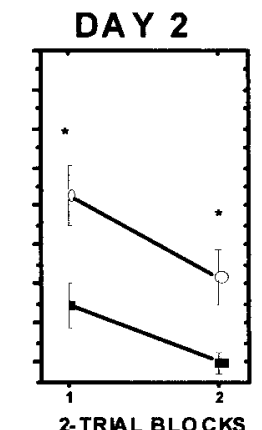

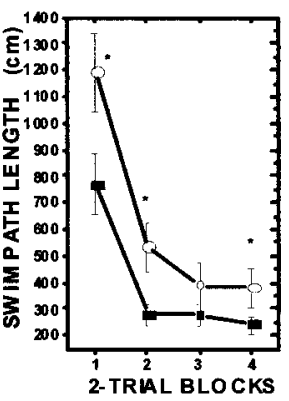

Figure 2. A, Mean escape latencies in a water maze during place-training trials in 18 rats exposed to EHYP for $14 \mathrm{~d}(\bigcirc)$ and 19 control rats ( $)$ on days 1 and 2 of the trials (*, EHYP vs control, $p<0.01$, ANOVA). $B$, Mean swim path lengths in a water maze during place-training trials in 18 rats exposed to EHYP for $14 \mathrm{~d}(\bigcirc)$ and 19 control rats $(\mathbf{\square})$ on days 1 and 2 of the trials (*, EHYP vs control, $p<0.01$, ANOVA). $C$, Mean escape latencies in a water maze during cued trials (right) and mean target quadrant times during probed trials $(l e f t)$ in 18 rats exposed to EHYP for $14 \mathrm{~d}(\mathrm{O}$, right; open column, left $)$ and 19 control rats (ם, right; filled column, left) (EHYP vs control, NS). D, Mean escape latencies (left) and swim path lengths (right) in a water maze during place-training trials in 13 rats exposed to EHYP for $14 \mathrm{~d}(\mathrm{O})$ and 13 control rats $(\mathbf{\square})$ tested after $14 \mathrm{~d}$ of normoxic recovery on days 1 and 2 of the trials (*, EHYP vs control, $p<0.01$, ANOVA).

\section{Immunohistochemistry: NMDA-c-fos immunoreactivity}

The expression of NMDA glutamate receptor was examined in double-labeling experiments for NR1 and c-fos immunoreactivities in eight rats exposed to EHYP and in eight control animals. Disruption of the hippocampal CA1 cellular arrangement with enhanced c-fos expression occurred in all eight EHYP-exposed rats (Figs. 3, 4). Similarly, marked reductions in the number of neurons harboring NMDA NR1 labeling occurred in the cortex $(\mathrm{Cx})$ and $\mathrm{CA} 1$ region. In the cortex, c-fos protein was primarily expressed within small cells, which were most likely glia because they also stained for GFAP (Fig. 5). Within the CA1 region, enhanced c-fos immunoreactivity was restricted to NR1-positive cells. In marked contrast with the findings in these two regions, no apparent changes were readily identifiable in the CA3 hippocampal region. Further semiquantitative analyses of c-fos protein expression revealed that significant differences emerged in EHYP-exposed animals both for duration of exposure and regional distribution (CA1, CA3, and Cx; Fig. 4).

\section{Apoptosis}

\section{Single-stranded DNA}

The effects of EHYP on early induction of apoptosis as evidenced by increased immunoreactivity for single-stranded DNA (SSDNA) were examined in rats exposed to EHYP for 0, 1, 2, 7, and $14 \mathrm{~d}$. Although SS-DNA was rarely present in the hippocampus and neocortex of control animals (day 0), marked enhancements in the number of cells positive for SS-DNA became apparent in these structures at days 1 and 2 of EHYP and were followed by a reduction of such nuclear immunoreactivity at days 7 and 14 (Fig. $6)$. These changes were similar in the CA1 region and in the neocortex but were at variance in the CA3 region of the hippocampus where no significant changes occurred over time with EHYP (Fig. 7).

\section{TUNEL}

EHYP elicited significant enhancements in the number of cells displaying positive TUNEL labeling (an indicator of apoptosis and necrosis) in CA1 and Cx but not in CA3 (Fig. 8). The overall time course for such enhancements in TUNEL labeling was similar to that found in SS-DNA (Fig. 9). However, at $14 \mathrm{~d}$ of EHYP, the density of cells with TUNEL-positive labeling was still elevated compared with control ( $p<0.05$, ANOVA).

\section{DISCUSSION}

Daylight EHYP is associated with transient sleep deprivation followed by sleep recovery. However after 14 d of EHYP, learning impairments during acquisition of a spatial memory task occurred and were only partially reversed after 14 d of recovery. Anatomical correlates for such decreases in behavioral maze performance show time-dependent increases in apoptosis within the cortex and CA1 region of the hippocampus with little effect on the CA3 region. The increase in cell loss within this region is associated with marked changes in the tissue distribution of NMDA-harboring cells within the affected regions and increased c-fos protein expression in surviving cells.

\section{Episodic hypoxia profile}

The selected EHYP profiles aimed to reproduce the overall cumulative hourly oxygen desaturation patterns routinely observed in moderately severe OSA patients. The cumulative arterial blood gases at various time points during the alternating $10 \%$ $\mathrm{O}_{2}$-room air $90 \mathrm{sec}$ cycle confirmed the anticipated alternation of moderate hypoxemia with normoxemia, such that calculated oxyhemoglobin saturations ranged from 62 to $70 \%$ and were clearly within the range of those recorded in OSA patients.

Other investigators have previously used similar EHYP approaches to mimic the episodic hypoxia of OSA. In these profiles, hypoxic cycles varied between $30 \mathrm{sec}$ (Fletcher, 1995; Sica et al., 2000) and 45 min (Chaufour et al., 1999). However, sleep architecture was not assessed. Notwithstanding such an important limitation, EHYP induced the increases in sympathetic outflow and the systemic and pulmonary artery pressures that typically occur in OSA patients (Bakehe et al., 1996; Lesske et al., 1997; 

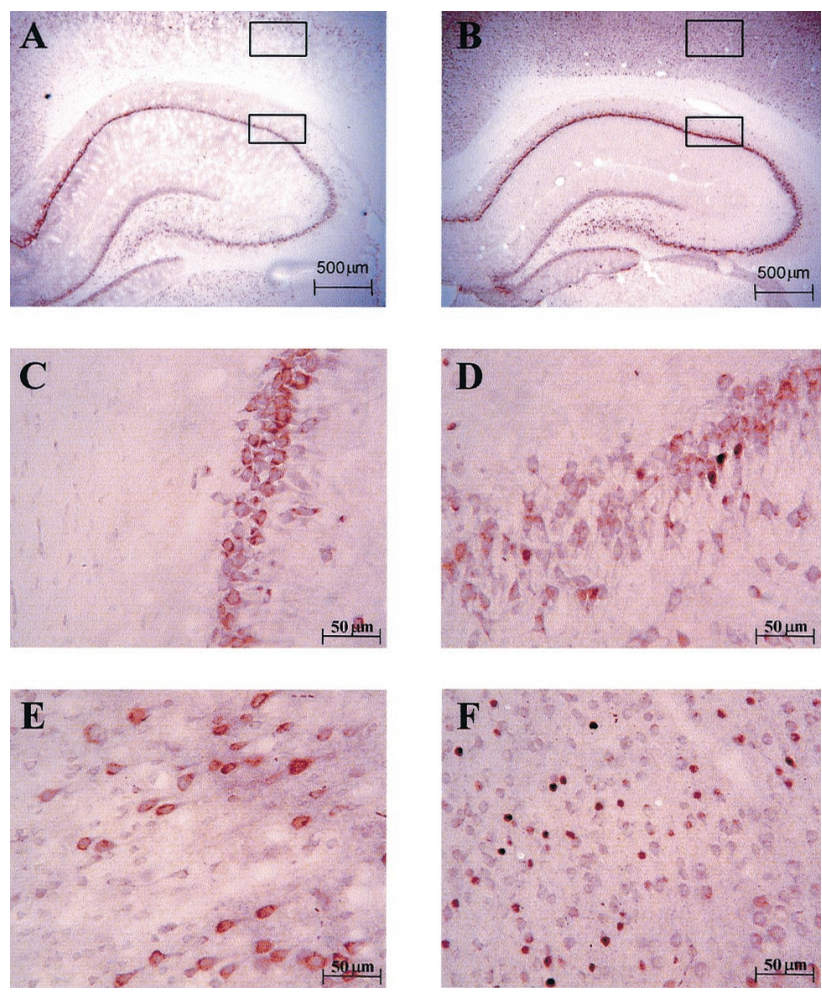

Figure 3. $A, B$, Photomicrographs of coronal sections through the hippocampal formation illustrating NR1 and c-fos immunoreactivity in room air $(A)$ and after EHYP for $14 \mathrm{~d}(B)$. $C, D$, Higher magnification of photomicrographs of the CA1 hippocampal region (lower boxed area in $A$, $B$, respectively) illustrating the dense NR1 labeling in this region and the relative paucity of c-fos-labeled neurons during control conditions $(C)$. In contrast, marked architectural disorganization is apparent in the CA1 region after a $14 \mathrm{~d}$ exposure to EHYP, and c-fos labeling is also more prominent and colocalizes with NR1-labeled pyramidal neurons $(D)$. E, $F$, Higher magnification of photomicrographs of the cortex (upper boxed area in $A, B$, respectively) illustrating abundant NR1 cellular labeling in this region $(E)$. After $14 \mathrm{~d}$ of EHYP, NR1-immunoreactive cells are scarce, and instead small cells (possibly glia) emerge and display enhanced c-fos nuclear expression $(F)$. The scale bar is shown on every image in the right-hand bottom corner.

McGuire and Bradford, 1999). Furthermore, increased c-fos labeling was seen in brainstem regions involved in the regulation of sympathetic neural discharge (Greenberg et al., 1999). The evolution of sleep architecture during EHYP indicated that the initial circadian phase of EHYP was associated with significant REM and NREM fragmentation. However, sleep recovery occurred, and the overall sleep architecture normalized thereafter. Thus, the rodent exposure model used herein provides initial insights into the regulation of sleep homeostasis during EHYP and offers the opportunity to examine selectively particular aspects of neurobehavioral and structural consequences of EHYP exposure during sleep.

\section{Neurobehavioral studies}

The present experiments indicate that $14 \mathrm{~d}$ of EHYP impaired acquisition of a water maze task that depended on spatial reference memory. Males exposed to EHYP performed significantly more poorly than did control males on place-training trials across $2 \mathrm{~d}$ of testing. There were no effects of EHYP on performance during cued trials, indicating that all rats could discriminate and

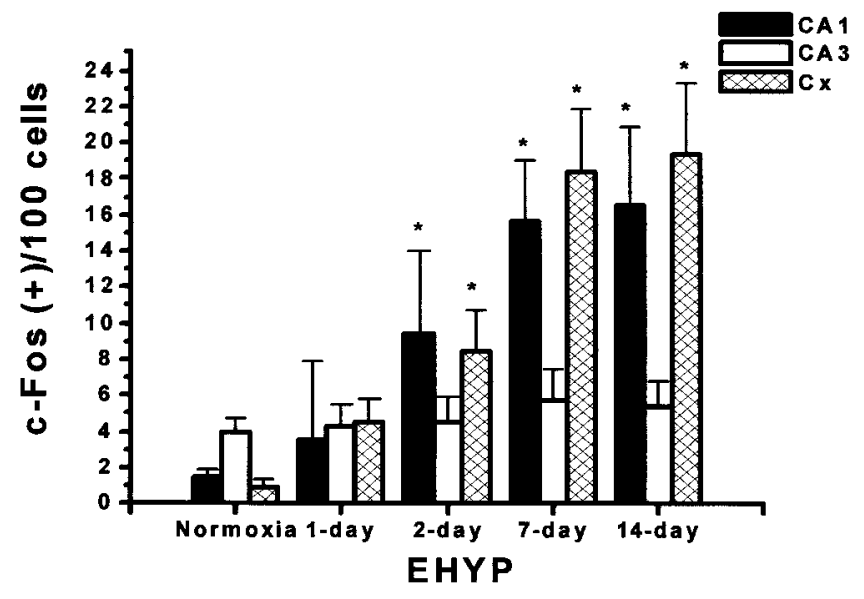

Figure 4. Mean $( \pm$ SD) number of c-fos-positive cells per 100 cells in the CA1 region (filled columns) and CA3 region (open columns) of the hippocampus and the adjacent cortex (cross-hatched columns). Significant increases in c-fos (shown as ${ }^{*}$ ) occurred with EHYP in all regions over time (vs control: CA1, $p<0.0001$, ANOVA; CA3, $p<0.03$, ANOVA; $\mathrm{Cx}$, $p<0.0001$, ANOVA $)$ and were more prominent in CA1 and $\mathrm{Cx}(p<$ 0.001 vs CA3) ( $n=8$ rats for each time point; normoxia indicates control rats).
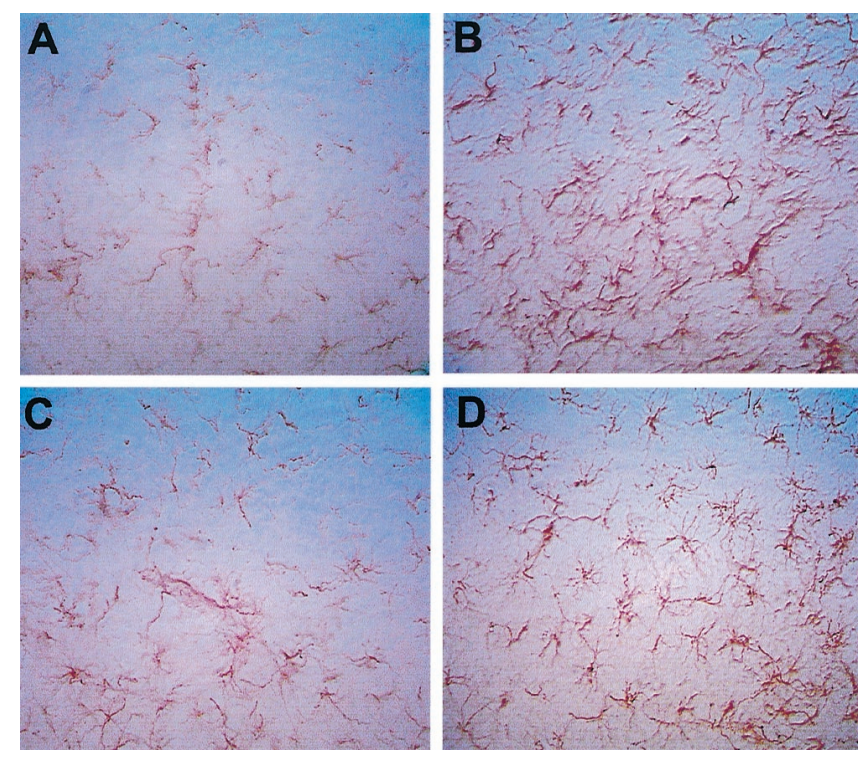

Figure 5. Photomicrographs of coronal cortical sections illustrating GFAP immunoreactivity in two control rats $(A, C)$, and in two rats after exposure to EHYP for $14 \mathrm{~d}(B, D)$.

swim equally well to a marked escape platform. Unexpectedly, there were no significant differences revealed between the groups during a single-probe trial to measure retention that may have been inadequate to detect a spatial bias for the target quadrant. However, after $14 \mathrm{~d}$ of recovery, males exposed to EHYP continued to perform more poorly than did males exposed to normoxia on place-training trials, although the difference in performance was much less than was demonstrated on place-training trials conducted on the days immediately after termination of hypoxic conditions.

EHYP may affect water maze performance by altering various nonmnemonic factors rather than the neural substrates that directly mediate learning and memory. These could include body 
a

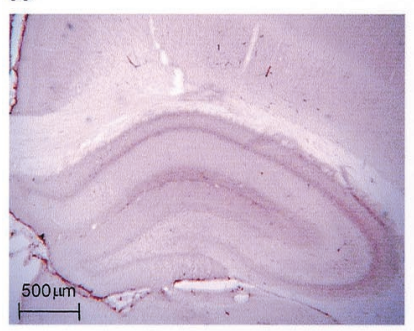

b

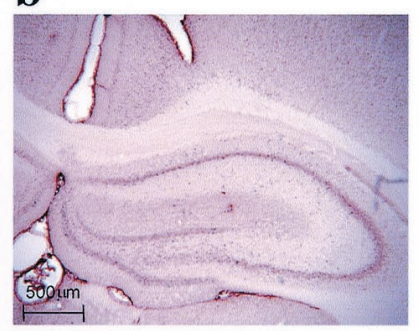

c

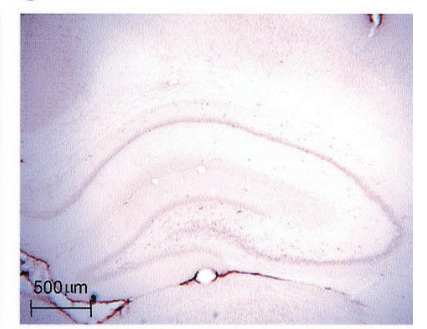

d

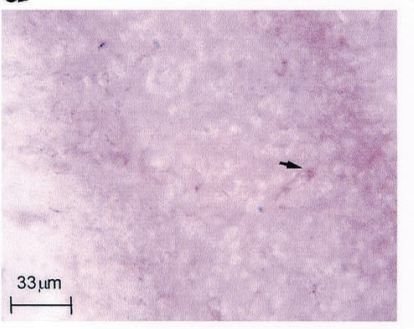

e

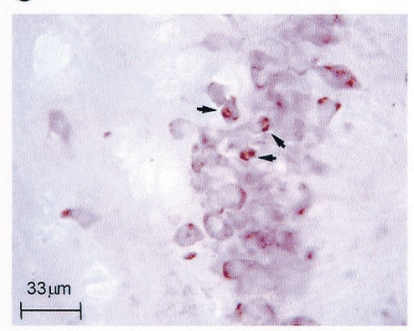

f
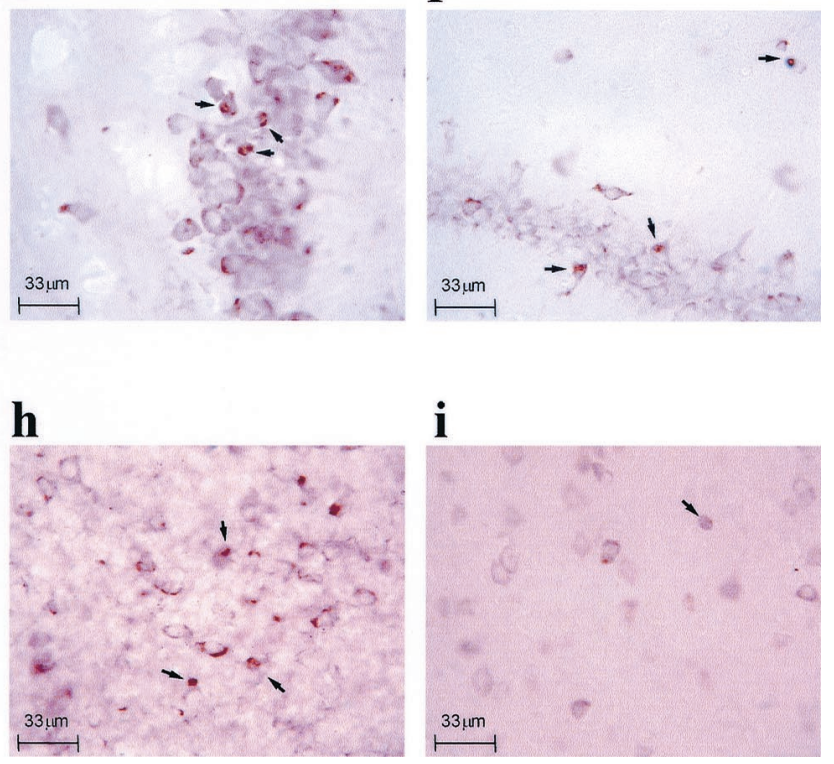

i

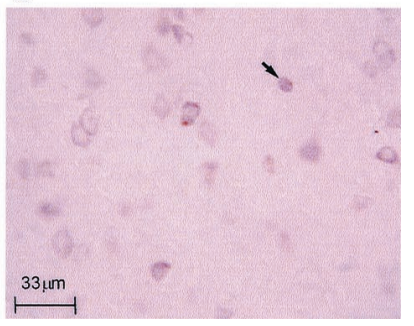

Figure 6. $a-c$, Photomicrographs of coronal sections through the hippocampal formation illustrate SS-DNA immunoreactivity in room air $(a)$ and after EHYP for $2 \mathrm{~d}(b)$ and $7 \mathrm{~d}$ (c). $d-f$, Higher magnification of photomicrographs of the CA1 hippocampal region illustrates the scarce SS-DNA labeling in this region during normoxic conditions $(d)$ and the marked enhancements in SS-DNApositive cells at $2 \mathrm{~d}$ EHYP $(e)$, followed by some reduction at $7 \mathrm{~d}$ EHYP $(f)$. $g-i$, Similarly, neocortical regions exhibited only occasional SS-DNA nuclear staining in control $(g)$ but marked enhancements after EHYP at $2 \mathrm{~d}$ $(h)$ and reductions in SS-DNA labeling at $7 \mathrm{~d}$ (i). The scale bar is shown on every image in the left-hand bottom corner.

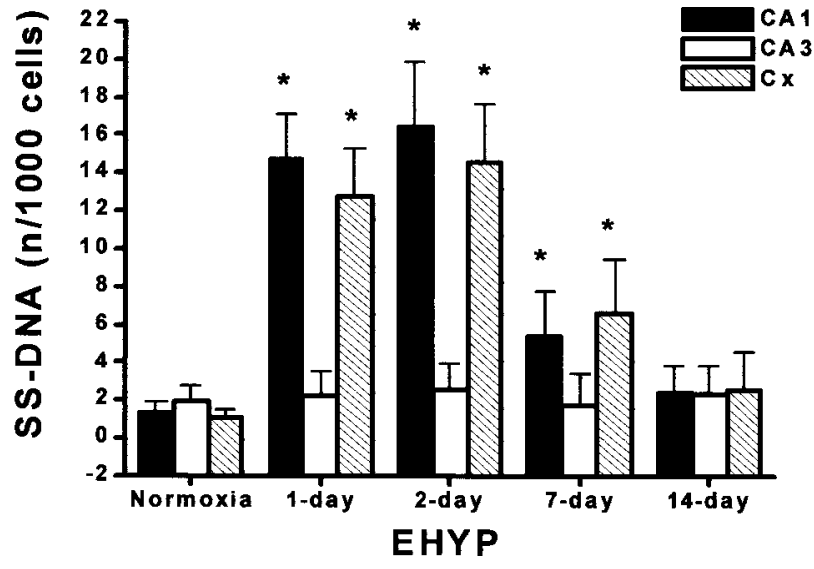

Figure 7. Mean $( \pm$ SD) number of SS-DNA-positive cells per 1000 cells in the CA1 region ( filled columns) and CA3 region (open columns) of the hippocampus and the adjacent cortex (hatched columns). Significant increases in SS-DNA labeling occurred with EHYP in CA1 $(p<0.001$ vs time 0$)$ and $\mathrm{Cx}(p<0.001$ vs time 0$)$ but not in CA3 (NS vs time 0$)$. A biphasic pattern emerged, by which SS-DNA labeling peaked at $24-48 \mathrm{hr}$ and returned to baseline levels after $14 \mathrm{~d}$ of $\operatorname{EHYP}(n=4$ rats for each time point; ${ }^{*} p<0.05$ vs time 0 ).

weight, sensorimotor function, thermoregulation, and fear and stress responses (Albert et al., 1970; Hicks and Moore, 1979; Ettinger and Staddon, 1982; Horne and McGrath, 1984; ShukittHale et al., 1994; Mabry et al., 1996; Youngblood et al., 1997).
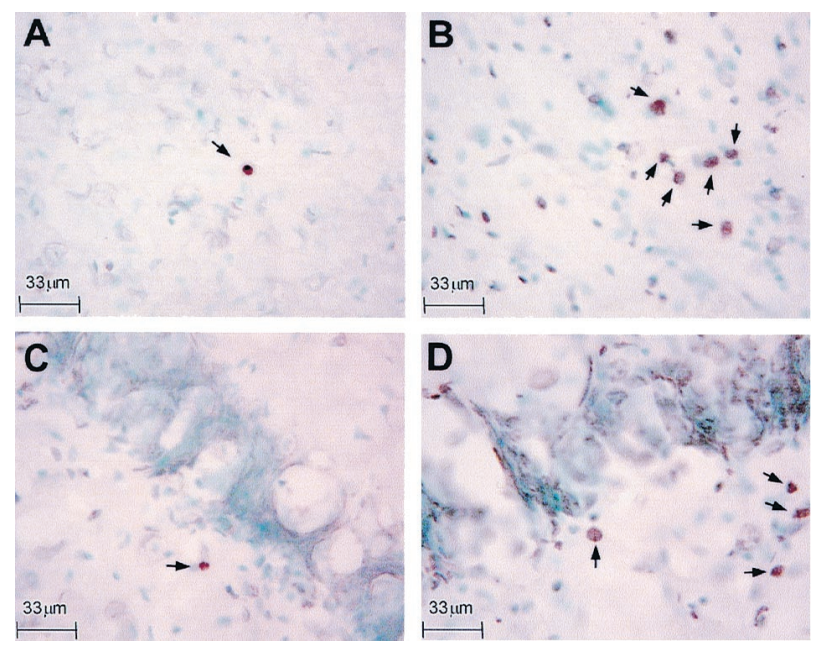

Figure 8. Photomicrographs of coronal sections of neocortical regions illustrating TUNEL labeling counterstained with methyl green, in a rat exposed to room air $(A)$ and after EHYP for $2 \mathrm{~d}(B)$. Similarly, the CA1 region of the hippocampal formation exhibited only occasional labeling in a control animal $(C)$ but marked enhancements after $2 \mathrm{~d}$ of EHYP $(D)$. The scale bar is shown on every image in the left-hand bottom corner. Examples of positive TUNEL-labeled cells are indicated by the arrows.

However, in the present study, no significant differences were found between EHYP-exposed rats and normoxic rats on the first two place-training trials or during cued trials, indicating that all rats could swim equally well to the platform. Also, in addition to 


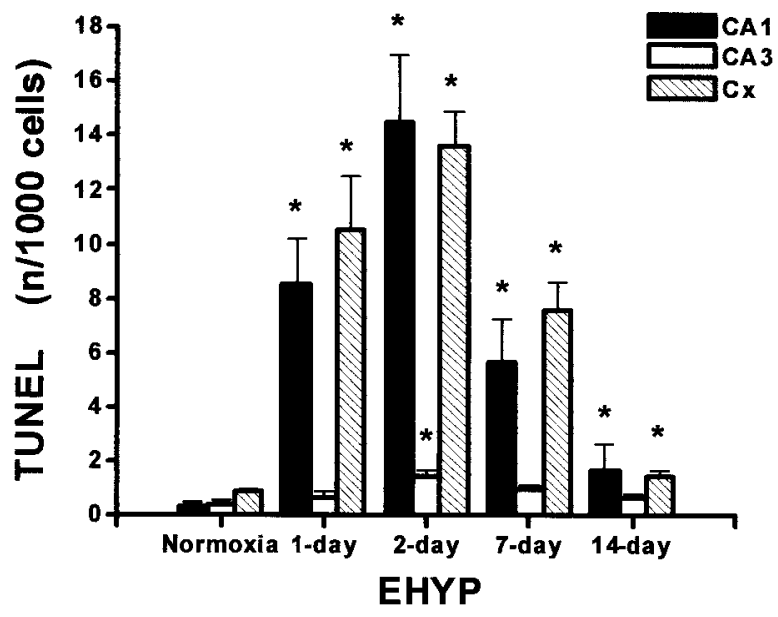

Figure 9. Mean $( \pm \mathrm{SD})$ number of TUNEL-positive cells per 1000 cells in the CA1 region ( filled columns) and CA3 region (open columns) of the hippocampus and the adjacent cortex (hatched columns). Significant increases in TUNEL labeling occurred with EHYP in CA1 $(p<0.001$ vs time 0$)$ and $\mathrm{Cx}(p<0.001$ vs time 0$)$, and smaller increases occurred at $2 \mathrm{~d}$ of EHYP in CA3 ( $p<0.05$ vs time 0$)$. A biphasic pattern emerged, such that TUNEL labeling peaked at $48 \mathrm{hr}$ and decreased thereafter, but without returning to baseline values at $14 \mathrm{~d}(n=4$ rats for each time point; ${ }^{*} p<0.05$ vs time 0 ).

latency to mount the platform, performance was measured by swim path length, a measure that was independent of swim speed. Thus, it is unlikely that nonmnemonic factors played a significant role in the impairment in performance seen in EHYP rats.

The ability to use spatial information from extramaze cues that was required in the reference memory version of the water maze task has been shown to be hippocampal dependent (Eichenbaum et al., 1990). Our results demonstrated that EHYP-exposed rats displayed marked cellular disruptions in the CA1 region of the hippocampus. Thus, it is not surprising that exposure to EHYP impaired performance during place-training trials but not during the cued trials (Morris, 1981, 1984). In fact, rats with hippocampal lesions were not impaired in the cued platform version of the water maze (Eichenbaum et al., 1990).

The behavioral analysis indicates that $14 \mathrm{~d}$ of EHYP impaired normal acquisition of a standard water maze task that depends on the hippocampus without affecting sensorimotor functions that are regulated by brain regions outside the hippocampus. After $14 \mathrm{~d}$ of recovery, rats exposed to EHYP continued to show a partial deficit in maze performance when the hidden platform was moved to a different quadrant. Interestingly, the partial recovery of cognitive deficits exhibited by EHYP-exposed rats is uniquely reminiscent of the persistence of short-term memory impairments in OSA patients despite effective therapy (Bedard et al., 1993; Naegele et al., 1998).

\section{NMDA-c-fos immunoreactivity}

The overall patterns of NR1 expression in the hippocampal and cortical regions of control animals are in close agreement with those of previous studies (Rigby et al., 1996; Rudolf et al., 1996). The marked alteration in cytoarchitectural boundaries within the pyramidal cell layer of CA1 and within the cortex is also compatible with the significant reductions in maze performance exhibited by EHYP-treated rats (Morris, 1989). Of note, extensive glial proliferation was apparent within the cortex but not within CA1 as demonstrated by GFAP immunoreactivity. However, the possible mechanism accounting for the disparity in glial response between these two EHYP-susceptible sites remains unclear. The reduction of NR1-positive cell density in the cortex and CA1 parallels the reduction in NMDA-binding sites reported by $\mathrm{Pi}$ chiule et al. (1996) in young rats chronically exposed to hypobaric hypoxia. Indeed, the cortex and hippocampus had a 36 and 35\% reduction in binding sites, respectively (Pichiule et al., 1996). Thus, long-term hypoxic conditions are associated with profound decrements in NMDA receptor expression, suggesting that NMDA glutamate receptor-expressing cells may have increased vulnerability to hypoxia, possibly via slowly evolving excitotoxicity processes (Albin and Greenamyre, 1992).

The marked increase in c-fos immunoreactivity after $14 \mathrm{~d}$ of EHYP exposure was not completely unexpected because such increases were shown previously within brainstem regions underlying autonomic regulation (Greenberg et al., 1999). This study further extends these observations to more rostral brain structures, but the significance of c-fos enhancements in the context of long-term EHYP exposure remains unknown. Of interest, increased c-fos immunoreactivity was primarily restricted to NR1immunoreactive neurons, and such a relationship may suggest an important role for immediate early genes in long-term survival and adaptation to stressful conditions such as intermittent oxygen deprivation or alternatively may indicate which neurons will irreversibly commit to apoptosis (Takemoto et al., 1995; Walton et al., 1998).

\section{Apoptosis}

Increased apoptotic staining occurred within the CA1 region of the hippocampus and cortex and followed a biphasic response by which after peak increases in apoptosis at 24-48 hr of EHYP, programmed cell death was gradually reduced and reached control values at $14 \mathrm{~d}$ for SS-DNA, while remaining slightly above control values for TUNEL. These data suggest that after exposure to EHYP, apoptotic mechanisms are induced and selectively localize to particular cellular populations within the hippocampal formation and cortex. The slight disparities between TUNEL and SS-DNA labeling at day 14 of EHYP could reflect sensitivity differences between the two methods in the detection of apoptosis. Alternatively, because TUNEL is considered to be a less specific marker of apoptosis (Grasl Kraupp et al., 1995; Frankfurt et al., 1996), other cells in the process of death by nonapoptotic mechanisms may have also been labeled (Chen et al., 1997; Labat Moleur et al., 1998). It needs to be emphasized that the magnitude of tissue hypoxia used in this study was relatively mild compared with the levels of hypoxia required for induction of apoptosis using a single exposure (Banasiak and Haddad, 1998). It is possible that the cumulative duration of hypoxia over time and/or the recurring pattern of the hypoxic challenge leading to hypoxia-reoxygenation injury may have exacerbated the susceptibility of more vulnerable cellular populations to EHYP insult.

The selective tolerance of the CA3 hippocampal region to EHYP is not surprising because of previous studies demonstrating the high susceptibility of CA1 to hypoxia when compared with CA3 (Kawasaki et al., 1990; Kreisman et al., 2000). Although the current experiments do not provide additional insights into the mechanisms underlying the selective regional vulnerability to EHYP, they provide the initial description of such phenomenon in the context of episodic hypoxia and suggest that cognitive functions primarily mediated by CA 1 rather than by CA 3 are 
more likely to be damaged. Furthermore, the apoptotic events described herein appear to be temporally restricted, suggesting either that all EHYP-sensitive cells within a region have been affected within a few days and undergone cell death or that some adaptive mechanisms may have been activated during the initial phases of EHYP to ensure long-term survival. Additional studies are clearly needed to elucidate which survival mechanisms confer tolerance to EHYP.

A major and obvious limitation of the present study is the descriptive nature of its findings. Although the present experiments provide an extensive characterization of a rodent model of intermittent hypoxia during sleep that is not associated with major sleep disruption, the mechanisms responsible for the neurobehavioral performance deficits, increased apoptosis, and cellular rearrangements within the hippocampal formation and neighboring cortical regions remain to be defined. Notwithstanding such limitations, the present model provides initial support for the conceptual framework that intermittent hypoxia during sleep is associated with substantial deterioration of behavioral performance and with parallel disruption of the anatomical substrate. Thus, the present model opens the way for future exploration of the individual roles and potential interactions between sleep disruption and intermittent hypoxia and hypercapnia and their roles in the pathophysiology of OSA-induced neurocognitive morbidity.

\section{REFERENCES}

Albert I, Cicala GA, Siegel J (1970) The behavioral effects of REM sleep deprivation in rats. Psychophysiology 6:550-560.

Albin RL, Greenamyre JT (1992) Alternative excitotoxic hypotheses. Neurology 42:733-738.

Bakehe M, Hedner J, Dang T, Chambille B, Gaultier CL, Escourrou P (1996) Role of the autonomic nervous system in the acute blood pressure elevation during repetitive hypoxic and hypercapnic breathing in rats. Blood Press 5:371-375.

Banasiak KJ, Haddad GG (1998) Hypoxia-induced apoptosis: effect of hypoxic severity and role of p53 in neuronal cell death. Brain Res 797:295-304.

Bedard MA, Monplaisir J, Richer F, Rouleau I, Malo J (1991) Obstructive sleep apnea syndrome: pathogenesis of neuropsychological deficits. J Clin Exp Neuropsychol 13:950-964.

Bedard MA, Monplaisir J, Malo J, Richer F, Rouleau I (1993) Persistent neuropsychological deficits and vigilance impairment in sleep apnea syndrome after treatment with continuous positive airways pressure (CPAP). J Clin Exp Neuropsychol 15:330-341.

Berry DT, Webb WB, Block AJ, Bauer RM, Switzer DA (1986) Nocturnal hypoxia and neuropsychological variables. J Clin Exp Neuropsychol 8:229-238.

Chaufour X, Issa F, Sullivan C, Mclachlan C, Unger G (1999) A fullyautomated environmental chamber for examination of long-term effects of intermittent hypoxia on medium-size animals. Jpn J Physiol 49:207-211.

Chen J, Jin K, Chen M, Pei W, Kawaguchi K, Greenberg DA, Simon RP (1997) Early detection of DNA strand breaks in the brain after transient focal ischemia: implications for the role of DNA damage in apoptosis and neuronal cell death. J Neurochem 69:232-245.

Eichenbaum H, Stewart C, Morris RGM (1990) Hippocampal representation in place learning. J Neurosci 10:3531-3542.

Ettinger RH, Staddon JER (1982) Decreased feeding associated with acute hypoxia in rats. Physiol Behav 29:455-458.

Fletcher EC (1995) An animal model of the relationship between systemic hypertension and repetitive episodic hypoxia as seen in sleep apnea. J Sleep Res 4:71-77.

Frank MG, Heller HC (1997) Development of diurnal organization of EEG slow activity and slow wave sleep in the rat. Am J Physiol 273:R472-R478.

Franken P, Dijk DJ, Tobler I, Borbely AA (1991) Sleep deprivation in the rat: effects on EEG power spectra, vigilance states, and cortical temperature. Am J Physiol 261:R198-R208.

Frankfurt OS, Robb JA, Sugarbaker EV, Villa L (1996) Monoclonal antibody to single-stranded DNA is a specific and sensitive marker of apoptosis. Exp Cell Res 226:387-397.

Gozal D (1998) Sleep-disordered breathing and school performance in children. Pediatrics 102:616-620.

Gozal D, Xue YD, Simakajornboon N (1999) Hypoxia induces c-fos protein expression in NMDA but not AMPA glutamate receptor labeled neurons within the nucleus tractus solitarii of the conscious rat. Neurosci Lett 262:93-96.

Grasl Kraupp B, Ruttkay Nedecky B, Koudelka H, Bukowska K, Bursch W, Schulte Hermann R (1995) In situ detection of fragmented DNA (TUNEL assay) fails to discriminate among apoptosis, necrosis, and autolytic cell death: a cautionary note. Hepatology 21:1465-1468.

Greenberg HE, Sica AL, Scharf SM, Ruggiero DA (1999) Expression of c-fos in the rat brainstem after chronic intermittent hypoxia. Brain Res 816:638-645.

Hicks RA, Moore JD (1979) REM sleep deprivation diminishes fear in rats. Physiol Behav 22:689-692.

Horne JA, McGrath MJ (1984) The consolidation hypothesis for REM sleep function: stress and other confounding factors-a review. Biol Psychol 18:165-184.

Kales A, Caldwell AB, Cadieux RJ, Vela-Bueno A, Ruch LG, Mayes SD (1985) Severe obstructive sleep apnea. II. Associated psychopathology and psychosocial consequences. J Chronic Dis 38:427-434.

Kawasaki K, Traynelis SF, Dingledine R (1990) Different responses of CA1 and CA3 regions to hypoxia in rat hippocampal slice. J Neurophysiol 63:385-394.

Kreisman NR, Soliman S, Gozal D (2000) Regional differences in hypoxic depolarization and swelling in hippocampal slices. J Neurophysiol 83:1031-1038.

Krueger JM, Kapás L, Kimura M, Opp MR (1993) Somnogenic cytokines: methods and overview. In: Neurobiology of cytokines (DeSouza EB, ed), pp 111-129. San Diego: Academic.

Labat Moleur F, Guillermet C, Lorimier P, Robert C, Lantuejoul S, Brambilla E, Negoescu A (1998) TUNEL apoptotic cell detection in tissue sections: critical evaluation and improvement. J Histochem Cytochem 46:327-334.

Lesske J, Fletcher EC, Bao G, Unger T (1997) Hypertension caused by chronic intermittent hypoxia-influence of chemoreceptors and sympathetic nervous system. J Hypertens 15:1593-1603.

Mabry TR, Mccarty R, Gold PE, Foster TC (1996) Age and stress history effects on spatial performance in a swim task in Fischer-344 rats. Neurobiol Learn Mem 66:1-10.

McGuire M, Bradford A (1999) Chronic intermittent hypoxia increases haematocrit and causes right ventricular hypertrophy in the rat. Respir Physiol 117:53-58.

Morisson F, Lavigne G, Petit D, Nielsen T, Malo J, Montplaisir J (1998) Spectral analysis of wakefulness and REM sleep EEG in patients with sleep apnea syndrome. Eur Respir J 11:1135-1140.

Morris RGM (1981) Spatial localisation does not require the presence of local cues. Learn Motiv 12:239-260.

Morris RGM (1984) Developments of a water-maze procedure for studying spatial learning in the rat. J Neurosci Methods 11:47-60.

Morris RGM (1989) Synaptic plasticity and learning: selective impairment of learning in rats and blockade of long-term potentiation in vivo by the $N$-methyl-D-aspartate receptor antagonist AP5. J Neurosci 9:3040-3057.

Morris RGM, Hagan JJ, Rawlins JNP (1986) Allocentric spatial learning by hippocampectomised rats: a further test of the spatial mapping and working memory theories of hippocampal function. Q J Exp Psychol 38:365-395.

Naegele B, Pepin JL, Levy P, Bonnet J, Pellat J, Feuerstein C (1998) Cognitive executive dysfunction in patients with obstructive sleep apnea syndrome (OSAS) after CPAP treatment. Sleep 21:392-397.

National Heart, Lung, Blood Institute Working Group on Sleep Apnea (1996) Sleep apnea: is your patient at risk? Am Fam Physician 53:247-253

Partinen M, Telakivi T (1992) Epidemiology of obstructive sleep apnea syndrome. Sleep 15:S1-S4.

Paxinos G, Watson C (1986) The rat brain in stereotaxic coordinates. New York: Academic.

Petralia RS, Yokotani N, Wenthold RJ (1994) Light and electron microscope distribution of the NMDA receptor subunit NMDAR1 in the rat nervous system using a selective anti-peptide antibody. J Neurosci 14:667-696.

Pichiule P, Chavez JC, Boero J, Arregui A (1996) Chronic hypoxia induces modification of the $N$-methyl-D-aspartate receptor in rat brain. Neurosci Lett 218:83-86.

Poceta JS, Timms RM, Jeong DU, Ho SL, Herman MK, Mitler MM (1992) Maintenance of wakefulness test in obstructive sleep apnea syndrome. Chest 101:893-897.

Redline S, Kump K, Tishler PV, Browner I, Ferrette V (1994) Gender differences in sleep disordered breathing in a community-based sample. Am J Respir Crit Care Med 149:722-726.

Rigby M, Le Bourdelles B, Heavens RP, Kelly S, Smith D, Butler A, Hammans R, Hills R, Xuereb JH (1996) The messenger RNAs for the $N$-methyl-D-aspartate receptor subunits show region-specific expression of different subunit composition in the human brain. Neuroscience 73:429-447.

Roehrs T, Merrion M, Pedrosi B, Stepanski E, Zorick F, Roth T (1995) 
Neuropsychological function in obstructive sleep apnea syndrome (OSAS) compared to chronic obstructive pulmonary disease. Sleep 18:382-388.

Rudolf GD, Cronin CA, Landwehrmeyer GB, Standaert DG, Penney JB, Young AB (1996) Expression of $N$-methyl-D-aspartate glutamate receptor subunits in the prefrontal cortex of the rat. Neuroscience 73:417-427.

Shukitt-Hale B, Stillman MJ, Welch DI, Levy A, Devine JA, Lieberman HR (1994) Hypobaric hypoxia impairs spatial memory in an elevation-dependent fashion. Behav Neural Biol 62:244-252.

Sica AL, Greenberg HE, Ruggiero DA, Scharf SM (2000) Chronicintermittent hypoxia: a model of sympathetic activation in the rat. Respir Physiol 121:173-184.
Takemoto O, Tomimoto H, Yanagihara T (1995) Induction of c-fos and c-jun gene products and heat shock protein after brief and prolonged cerebral ischemia in gerbils. Stroke 26:1639-1648.

Timo-Iaria C, Negrao N, Schmidek WR, Hoshino KWR, Menezes CE, Da Rocha TL (1970) Phases and states of sleep in the rat. Physiol Behav 5:1057-1062.

Walton M, MacGibbon G, Young D, Sirimanne E, Williams C, Gluckman P, Dragunow M (1998) Do c-Jun, c-Fos, and amyloid precursor protein play a role in neuronal death or survival? J Neurosci Res 53:330-342.

Youngblood BD, Zhou J, Smagin GN, Ryan DH, Harris RBS (1997) Sleep deprivation by the flower pot technique and spatial reference memory. Physiol Behav 61:249-256. 